\title{
Hard truths about preoperative knee X-rays
}

\author{
Wai-Keong Mak, Hamid Rahmatullah Bin Abd Razak \\ Department of Orthopaedic Surgery, Sengkang General Hospital, Singapore, Singapore \\ Correspondence to: Asst. Prof. Hamid Rahmatullah Bin Abd Razak, MBBS, FRCSEd (Orth), FRCSGlasg (Tr\&Orth). Department of Orthopaedic \\ Surgery, Sengkang General Hospital, 110 Sengkang East Way, Singapore 544886, Singapore. Email: hamidrazak@gmail.com. \\ Comment on: van de Water RB, Leichtenberg CS, Nelissen RGHH, et al. Preoperative Radiographic Osteoarthritis Severity Modifies the Effect of \\ Preoperative Pain on Pain/Function After Total Knee Arthroplasty: Results at 1 and 2 Years Postoperatively. J Bone Joint Surg Am 2019;101:879-87.
}

Submitted Mar 19, 2020. Accepted for publication Apr 17, 2020.

doi: 10.21037/atm-20-2686

View this article at: http://dx.doi.org/10.21037/atm-20-2686

Osteoarthritis (OA) contributes significantly towards disease morbidity burden (1). Although total knee arthroplasty (TKA) is a successful treatment option for endstage OA, $15 \%$ to $25 \%$ of patients report dissatisfaction postoperatively (2). Therefore, efforts have been made to develop appropriate criteria for preoperative patient selection and prioritization for TKA. It is essential to find predictors of postoperative outcomes in patients undergoing TKA. Poorer preoperative function has been found to predict for poorer postoperative function (3). Preoperative radiographic classification and selfreported pain scores have also been reported as important factors in predicting success following TKA (4). On the other hand, there are issues inherent with diagnosis of OA with radiographs which confounds clinical practice. It is therefore timely and apt that van de Water et al. (5) conducted a multicentre prospective cohort study of 599 patients, evaluating the effects of preoperative radiographic OA severity on preoperative pain. Their article is entitled, "Preoperative Radiographic Osteoarthritis Severity Modifies the Effect of Preoperative Pain on Pain/Function After Total Knee Artbroplasty: Results at 1 and 2 Years Postoperatively".

In their study, the authors investigate if preoperative radiographical severity of $\mathrm{OA}$ correlated with preoperative self-reported pain, postoperative function and postoperative pain up to two years following surgery. Interestingly, the study found (I) better 1-year postoperative pain outcomes in patient with more severe OA seen on preoperative radiographs; (II) patients with higher preoperative pain levels can be expected to experience more pain following TKA and; (III) the effect of preoperative pain on postoperative pain and function seems to be less important when there was radiographic evidence of greater preoperative severity. They concluded that the effect modification of radiographically severity should be taken into account when new prognostic models for outcomes after TKA are established.

The authors reported that patients with milder radiographic OA were likely to be operated on as a result of their pain rather than radiographic OA severity, as confirmed by similar baseline pain levels across all four different Kellgren-Lawrence groups. Sources of pain that may not be evident on radiographs include extra-articular pathologies, psychosomatic conditions (6) or central pain sensitization (5). Patients with higher pain scores and lower Kellgren-Lawrence grades demonstrated more central pain sensitization (7), which subsequently lead to less improvement following TKA compared with patients with high Kellgren-Lawrence grades and high pain levels (5). The effect of radiographical severity of OA and diffuse hyperalgesia effect on pre- and postoperative pain scores were previously also studied by Wylde et al. (8). They found that patients with milder OA severity and more diffuse hyperalgesia demonstrated less improvement in pain after TKA than patients with less diffuse hyperalgesia (8). However, only patients with 3 and 4 Kellgren-Lawrence grades were included in their study due to the small numbers of patients with Kellgren-Lawrence grades $\leq 2$. Therefore, their results need to be interpreted in light of this potential for selection bias. Patients with similar radiographical degeneration but multiple sources of preoperative pain may report different postoperative outcomes simply because other underlying causes for the pain maybe have not been resolved by surgery (5). This is also in concordance with 
a study that found patients with preoperative depression and anxiety to have increased preoperative pain and poorer postoperative outcomes following TKA (9). A discordance between observed radiographical severity and reported pain suggests that other factors may affect patient-perceived pain scores (10). In a study by Chang et al., it was found that severe spinal pathology was highly associated with poorer preoperative functional scores of patients undergoing TKA (11). They also reported that activity-related radiculopathy may herald poorer outcomes following TKA (11). In the context of clinical practice, it may be prudent to carefully consider other sources of knee pain in patients with low radiographical OA prior to offering TKA.

In their paper, van de Water et al. (5) report that effects of preoperative pain on postoperative pain and function seems to be less important when there was a radiographic evidence of greater arthritic severity. We believe this may be so as pain is no longer be the only limiting factor in patients with severe OA. Instead, other factors such preoperative stiffness may take on a greater role. Although TKA improved range of motion (ROM) and functional scores in patients with poor preoperative ROM (12), Lee et al. found that final ROM and clinical scores of patient with severe preoperative stiffness were inferior, with a higher complication rate than in the moderately stiff group (13). They found that even though these patients obtained substantial ROM gain after surgery, only a subset of patients ( $48 \%$ in the study) was able to achieve an ROM of $110^{\circ}$ flexion, which is needed to perform daily activities (14). Hence although TKA can improve ROM and functional outcomes in patients with severe stiffness, this may not be enough to fulfil expectations of these patients.

There are however inevitable issues inherent with diagnosis of OA with imaging as well as self-reported scores in OA and following TKA. Over recent years, several diagnostic modalities have been emerged. In spite of more advanced imaging techniques that are available today, traditional radiographs are still the most accessible modality to determine OA severity (15). The plain X-ray of the knee is usually used in diagnosing and monitoring the progression of OA (16). Yet previous articles have reported disagreement between radiological OA severity with functional scores (17). Despite the lack of correlation, guidelines still advocate the use of weight-bearing plain radiography in OA (15). The use of other imaging techniques such as magnetic resonance imaging (MRI), ultrasound, computed tomography (CT), and nuclear medicine in OA evaluation has been extensively studied with each posing its own set of limitations (18).
As such, we are heavily reliant upon the radiographic Kellgren-Lawrence grading system for diagnosis and monitoring of OA. Kellgren-Lawrence grading system has its disadvantages since it suggests a linear radiographic progression of $\mathrm{OA}$ and is incapable of quantifying joint space narrowing of the knee joint without osteophyte formation (19). Abdelaziz et al. found a significant mismatch between intraoperative and radiological findings with approximately $50 \%$ of the patients demonstrating grade 4 degenerative changes intraoperatively, compared to grade 3 radiographically (15). Of note, Kijowski et al. demonstrated that the Kellgren-Lawrence scoring system had only a moderately strong correlation with the actual degree of articular cartilage degeneration within the tibiofemoral joint (20). Although a survey study documented an agreement among orthopaedic surgeons that radiographic evidence of loss of joint space together with severe daily pain was an important indicator for TKA (4), joint space narrowing is not defined in the description of the KellgrenLawrence grading. This inaccuracy can lead to varying results of classification (21).

In addition, the scoring system deals with the joint as a whole, not paying attention to each joint compartment separately (15). A weak correlation was found between function and combined space narrowing of tibiofemoral and patellofemoral joints (22) whereas Reichenbach et al. revealed that radiographic evidence of bone attrition leads to functional disability (23). Chang et al. reported that varus mechanical alignment was the strongest predictor of greater pain and poorer function in patients with varus malalignment and advanced OA. They also proposed that this could be the primary indicator of objective disease severity when choosing interventional strategies for advanced knee OA (24). Although some studies agree that individual radiological features predict function and pain in knee OA, there is no agreement about the most important feature (25). A study by Dowsey et al. found a significant association between the preoperative function and lateral compartment osteophytes in patients with end-stage OA (25). Of note, the presence of residual posterior osteophytes in TKA patients was documented to be a significant predictor of postoperative knee flexion of $<90^{\circ}$ (26) while their removal was found to independently contribute to a better range of movement following TKA (27). The Kellgren and Lawrence scoring system does not account for these factors discussed above, in the staging of OA. While orthogonal knee radiographs are the cornerstone for radiological assessment, the possible variations in projections mean that findings need to be interpreted with 
great attention as suggested by Dowsey and colleagues (25).

Using the International Knee Society Score (IKS) as a validated scoring system for TKA (28), also poses its own set of limitations, as it is partially completed by the health care provider and therefore introducing observer bias. It was documented that IKS filled by orthopaedic surgeons or other healthcare staff, is liable to vary significantly from the patient's evaluation, in particular after a surgical procedure (29). In contrast, quality of life questionnaires, also called "self-assessment" questionnaires, filled by patients only, were demonstrated to be more objective (28), since they correspond more closely to patients' experience (30). However these are difficult to be utilized in routine clinical practice, even in their simplified version (31).

We commend van de Water et al. for the excellent work and discussion on the topic of preoperative radiographic OA severity modifying the effect of preoperative pain on pain and function following TKA. Dissatisfaction after TKA is a significant issue which needs to be addressed; $15 \%$ to $25 \%$ of patients report dissatisfaction with their improvement of physical function postoperatively (2). In this editorial, we discussed the caveats of using the Kellgren-Lawrence radiographic scoring system and its discordance with patient symptoms and functional scores. Despite this, it is clinically relevant and prudent for the Orthopaedic Surgeon to realize the significance of preoperative radiographic OA severity and its effect on patient outcomes.

\section{Acknowledgments}

Funding: None.

\section{Footnote}

Provenance and Peer Review: This article was commissioned and reviewed by the Academic Editor Dr. Liang Gao (Center of Experimental Orthopaedics, Saarland University Medical Center, Homburg/Saar, Germany).

Conflicts of Interest: Both authors have completed the ICMJE uniform disclosure form (available at http://dx.doi. org/10.21037/atm-20-2686). Dr. Bin Abd Razak serves as an unpaid editorial board member of Annals of Translational Medicine from Jul 2018 to Jun 2020. The other author has no conflicts of interest to declare.

Ethical Statement: The authors are accountable for all aspects of the work in ensuring that questions related to the accuracy or integrity of any part of the work are appropriately investigated and resolved.

Open Access Statement: This is an Open Access article distributed in accordance with the Creative Commons Attribution-NonCommercial-NoDerivs 4.0 International License (CC BY-NC-ND 4.0), which permits the noncommercial replication and distribution of the article with the strict proviso that no changes or edits are made and the original work is properly cited (including links to both the formal publication through the relevant DOI and the license). See: https://creativecommons.org/licenses/by-nc-nd/4.0/.

\section{References}

1. Mathers CD, Stevenson CE, Vos ET, et al. The Australian Burden of Disease Study: measuring the loss of health from diseases, injuries and risk factors. Med J Aust 2000;172:592-6.

2. Baker PN, Van der Meulen J, Lewsey J, et al. The role of pain and function in determining patient satisfaction after total knee replacement: data from the National Joint Registry for England and Wales. J Bone Joint Surg Br 2007;89:893-900.

3. Fortin PR, Penrod JR, Clarke AE, et al. Timing of total joint replacement affects clinical outcomes among patients with osteoarthritis of the hip or knee. Arthritis Rheum 2002;46:3327-30.

4. Mancuso CA, Ranawat CS, Esdaile JM, et al. Indications for total hip and total knee arthroplasties: results of orthopaedic surveys. J Arthroplasty 1996;11:34-46.

5. van de Water RB, Leichtenberg CS, Nelissen RG, et al. Preoperative Radiographic Osteoarthritis Severity Modifies the Effect of Preoperative Pain on Pain/Function After Total Knee Arthroplasty: Results at 1 and 2 Years Postoperatively. J Bone Joint Surg Am 2019;101:879-87.

6. Felson DT. The sources of pain in knee osteoarthritis. Curr Opin Rheumatol 2005;17:624-8.

7. Finan PH, Buenaver LF, Bounds SC, et al. Discordance between pain and radiographic severity in knee osteoarthritis: findings from quantitative sensory testing of central sensitization. Arthritis Rheum 2013;65:363-72.

8. Wylde V, Sayers A, Odutola A, et al. Central sensitization as a determinant of patients' benefit from total hip and knee replacement. Eur J Pain 2017;21:357-65.

9. Brander VA, Stulberg SD, Adams AD, et al. Ranawat Award Paper: Predicting total knee replacement pain: A prospective, observational study. Clin Orthop Relat Res 
2003;416:27-36.

10. Bedson J, Croft PR. The discordance between clinical and radiographic knee osteoarthritis: a systematic search and summary of the literature. BMC Musculoskelet Disord 2008;9:116.

11. Chang CB, Park KW, Kang YG, et al. Coexisting lumbar spondylosis in patients undergoing TKA: how common and how serious? Clin Orthop Relat Res 2014;472:710-7.

12. McAuley JP, Harrer MF, Ammeen D, et al. Outcome of knee arthroplasty in patients with poor preoperative range of motion. Clin Orthop Relat Res 2002:203-7.

13. Lee SA, Kang SB, Chang CB, et al. Does the severity or cause of preoperative stiffness affect the clinical results and range of motion after total knee arthroplasty? PLoS One 2018;13:e0205168.

14. Park KK, Chang CB, Kang YG, et al. Correlation of maximum flexion with clinical outcome after total knee replacement in Asian patients. J Bone Joint Surg Br 2007;89:604-8.

15. Abdelaziz H, Balde OM, Citak M, et al. KellgrenLawrence scoring system underestimates cartilage damage when indicating TKA: preoperative radiograph versus intraoperative photograph. Arch Orthop Trauma Surg 2019;139:1287-92.

16. Wright RW, Ross JR, Haas AK, et al. Osteoarthritis classification scales: interobserver reliability and arthroscopic correlation. J Bone Joint Surg Am 2014;96:1145.

17. Claessens A, Schouten J, Van den Ouweland F, et al. Do clinical findings associate with radiographic osteoarthritis of the knee? Ann Rheum Dis 1990;49:771-4.

18. Hayashi D, Roemer FW, Guermazi A. Imaging for osteoarthritis. Ann Phys Rehabil Med 2016;59:161-9.

19. Altman RD, Hochberg M, Murphy JW, et al. Atlas of individual radiographic features in osteoarthritis. Osteoarthritis Cartilage 1995;3:3-70.

20. Kijowski R, Blankenbaker D, Stanton P, et al. Arthroscopic validation of radiographic grading scales of osteoarthritis

Cite this article as: Mak WK, Bin Abd Razak HR. Hard truths about preoperative knee X-rays. Ann Transl Med 2020;8(18):1196. doi: 10.21037/atm-20-2686 of the tibiofemoral joint. AJR Am J Roentgenol 2006;187:794-9.

21. Schiphof D, de Klerk BM, Kerkhof HJ, et al. Impact of different descriptions of the Kellgren and Lawrence classification criteria on the diagnosis of knee osteoarthritis. Ann Rheum Dis 2011;70:1422-7.

22. Szebenyi B, Hollander AP, Dieppe P, et al. Associations between pain, function, and radiographic features in osteoarthritis of the knee. Arthritis Rheum 2006;54:230-5.

23. Reichenbach S, Dieppe PA, Nüesch E, et al. Association of bone attrition with knee pain, stiffness and disability: a cross-sectional study. Ann Rheum Dis 2011;70:293-8.

24. Chang CB, Koh IJ, Seo ES, et al. The radiographic predictors of symptom severity in advanced knee osteoarthritis with varus deformity. Knee 2011;18:456-60.

25. Dowsey MM, Dieppe P, Lohmander S, et al. The association between radiographic severity and preoperative function in patients undergoing primary knee replacement for osteoarthritis. Knee 2012;19:860-5.

26. Yau WP, Chiu KY, Tang WM, et al. Residual posterior femoral condyle osteophyte affects the flexion range after total knee replacement. Int Orthop 2005;29:375-9.

27. Ritter MA, Harty LD, Davis KE, et al. Predicting range of motion after total knee arthroplasty: clustering, log-linear regression, and regression tree analysis. J Bone Joint Surg Am 2003;85:1278-85.

28. Davies AP. Rating systems for total knee replacement. The Knee 2002;9:261-6.

29. Wright JG, Rudicel S, Feinstein A. Ask patients what they want. Evaluation of individual complaints before total hip replacement. J Bone Joint Surg Br 1994;76:229-34.

30. Jenny JY, Diesinger Y. Validation of a French version of the Oxford knee questionnaire. Orthop Traumatol Surg Res 2011;97:267-71.

31. Ware J Jr, Kosinski M, Keller SD. A 12-Item Short-Form Health Survey: construction of scales and preliminary tests of reliability and validity. Med Care 1996;34:220-33. 